\title{
How does the rat (Rattus norvegicus) adjust food-carrying responses to the influences of distance, effort, predatory odor, food size, and food availability?
}

\author{
IAN Q. WHISHAW and HANS C. DRINGENBERG \\ University of Lethbridge, Lethbridge, Alberta, Canada
}

\begin{abstract}
Foraging animals will behave in such a way that they maximize foraging efficiency while minimizing risk to predation. Using a behavioral paradigm in which laboratory rats (Rattus norvegicus) were provided with food at one end of an elevated beam and a refuge to which they could carry the food at the other end of the beam, we examined the effects of travel distance, travel difficulty, ambient lighting, predation risk, and food size on foraging behavior. Both lengthening travel distance (by increasing beam length) and increasing travel difficulty (by decreasing beam width) decreased food carrying. When plotted as a function of travel time, the effects of the two treatments appeared to be partly different. Decreasing ambient illumination decreased food carrying, although the rats still responded to distance and food size. In contrast, predator's odor (cat) stopped foraging altogether. When a store of small food pellets was available, the rats ate for prolonged periods in the open, and eating time increased with travel distance; however, when large food pellets were also available, the rats immediately selected and carried them to the refuge. The experiments show that food-carrying behavior in the rat is influenced by a variety of independently acting factors, which include eating time, food size, travel distance, travel risk, illumination, predation, and exposure time. The varied and flexible foraging behavior of the animals is discussed in relation to optimal foraging theory.
\end{abstract}

Foraging animals will trade off eating time against risk of exposure to competition or predation in such a way that eating is maximized and risk is minimized (see Krebs \& McCleery, 1984). This means that animals must be able to monitor and adjust their foraging behavior in order to respond to a wide range of influences, which may be additive or conflicting. Although the laboratory rat (Rattus norvegicus) has been used for the study of food-carrying behavior for more than $\mathbf{5 0}$ years, little examination has been made of the environmental factors that influence its behavior or the rules that the animal follows in dealing with variations in environmental conditions. A number of recent studies suggest, nevertheless, that its foraging behavior might be more complex than has been previously thought.

Food-carrying (hoarding) behavior of laboratory rats is influenced by food size. Foraging rats will eat small pieces of food at a food source but carry larger pieces of food to a refuge (Ewer, 1971; McNamara \& Whishaw, 1990; Phelps \& Roberts, 1989; Whishaw, Gorny, \& Dringenberg, 1991; Whishaw, Oddie, McNamara, Harris, \& Perry, 1990; Whishaw \& Tomie, 1989). This behavior both minimizes the time that the animals are exposed in the open

The authors thank the Canadian Natural Sciences and Engineering Research Council of Canada for supporting this research. Requests for reprints should be sent to Ian Q. Whishaw, Department of Psychology, University of Lethbridge, Lethbridge, Alberta T1K 3M4, Canada. (fax: 403-329-2057. e-mail: Whishaw@Hg.Uleth.Ca) and maximizes their food intake. Similar results have been obtained with the grey squirrel, Sciurus carolinensis (Lima \& Valone, 1986; Lima, Valone, \& Caraco, 1985), and the black-capped chickadee, Parus atricapillus (Lima, 1985) during foraging in natural environments. Examination of the stimulus properties of food that induce animals to carry suggests that anticipated eating time is important (Lima, 1985, 1987; Lima et al., 1985; Whishaw, 1990). A suggested rule is that food is carried when the time it will take to eat it exceeds return time to a refuge. Consistent with this suggestion, it has been found that for grey squirrels and chickadees, increases in travel distance proportionally decreased food carrying (Lima, 1985; Lima et al., 1985).

Part of the purpose of the present experiment was to examine whether distance similarly influences food carrying in rats. The question of the influence of travel distance is important because experiments performed to date suggest that animals measure distance in terms of travel time and thus base their decision to carry food on some ratio of travel time and eating time (Lima, 1985; Lima et al., 1985; Whishaw, 1990). Previous work, however, seemingly suggests that changes in distance do not influence rat food-carrying behavior (Whishaw \& Tomie, 1989). This previous result may have been obtained because the distances used were comparatively small. There are, however, species differences in the influence of distance. Valone and Lima (1987) report that 5 of 10 bird 
species examined failed to show decreases in food carrying as distance increased. Thus, it is possible that the rat is a species for which distance is not influential. Therefore, we examined the behavior of rats using a much larger range of distance than has been used previously.

An examination was also made of other factors that might influence food-carrying behavior. We hypothesized that if time was a critical underlying measure on which decisions to carry food were based, then no matter how travel time was varied, it should have a similar influence on food-carrying responses. This question was examined by varying travel distance on an elevated beam or holding distance constant and varying difficulty by varying beam width. Additional experiments examined the influences of apparent foraging risk by varying ambient lighting (Whishaw \& Oddie, 1989), by exposing the rats to the odor of a cat (Blanchard \& Blanchard, 1989) during foraging sessions, and by manipulating the size and quantity of food at the food source (Whishaw \& Tomie, 1989). These experiments were directed toward evaluating the degree to which rats can modify their behavior to cope with varying environmental and food-availability conditions.

\section{GENERAL METHOD}

\begin{abstract}
Animals
The animals used were 25 female rats (200-285 g) of the LongEvans strain. They were housed in hanging wire-mesh cages in groups of 5 animals per cage. The colony room was lighted on a 12:12-h schedule. Prior to testing, the rats were placed on a restricted feeding schectule that maintained them at approximately $90 \%$ normal body weight. In addition to the food that they received during the course of testing, they received a measured amount of food in the home cage once each day. Water was freely available.

\section{Food}

Rodent Chow food pellets (manufactured by Bioserve Inc., P.O. Box 450, Frenchtown, NJ) were used as food during the experiments. Pellets were of 10 different weights-20, 27, 45, 75, 94, $190,300,500,750$, and $1,000 \mathrm{mg}$ - which are referred to as sizes $1-10$, respectively.
\end{abstract}

\section{Data Collection}

In the experiments, food-handling behaviors and food-handling times were recorded. Behavioral events and their durations were entered, by manually operated microswitches, into an Apple Macintosh II Plus computer (Whishaw et al., 1990). The program allowed recording of the following food-handling behaviors: (1) eat, the rat takes a food pellet into its mouth and eats the food directly, (2) sit, food pellet is picked up by mouth and then transferred to the forepaws and is eaten while the rat adopts a sitting posture, and (3) carry, the food pellet is carried to the refuge where it is eaten. The following times were recorded: (1) eating time, time to eat a food pellet, (2) carry time, time to carry a pellet to the refuge after grasping it by mouth, (3) postprandial time, time spent in the refuge after eating the food pellet (Whishaw \& Gorny, 1991), and (4) return time, time taken to return to the food source after exiting the refuge.

Whenever a number of different-sized food pellets were given within a test session, presentation order was generated randomly by the computer program for each rat.

\section{EXPERIMENT 1 Effect of Travel Distance}

It has been demonstrated that some animals reduce food carrying as travel distance increases (Lima, 1985; Lima et al., 1985), whereas others do not (Valone \& Lima, 1978). Although a previous experiment with rats (Whishaw \& Tomie, 1989) reports that rat food carrying is not influenced by travel distance, the distances used in that study were relatively short. In the present study, the effect of travel distance on food carrying was reexamined using a wider range of distances. Five different distances were used, and the probability that rats would carry food pellets of different sizes was calculated for each distance.

\section{Method}

Apparatus. The testing apparatus consisted of a refuge cage attached to a beam, down which a rat could walk to obtain food. The refuge was a Plexiglas box $(30.5 \times 22.8 \times 22.8 \mathrm{~cm}$ high $)$, which had a door $(8 \mathrm{~cm}$ wide $\times 9.8 \mathrm{~cm}$ high) that gave access to the beam. The refuge was covered with black paper on all but one side. A video camera was pointed toward the open side and was connected to a monitor on which the rat and the inside of the cage could be viewed. The wooden beam was $8.3 \mathrm{~cm}$ wide and was supported by legs that held it $91 \mathrm{~cm}$ above the floor. Small holes were drilled into the beam at distances of $61,122,305,488$, and $671 \mathrm{~cm}$ away from the home cage. Food pellets were placed in the holes.

Pretraining. The rats were divided into five groups of 5 rats per group. The rats in each group were assigned to receive food at one of the five distances. During pretraining, the rats were placed in the cage in pairs and food pellets were placed at the appropriate target distance. Once the rats were retrieving the food from the beam, they were pretrained individually. At the end of about 2 weeks of pretraining, all of the rats would leave the cage and travel down the beam for food.

Testing. The rats were tested for 10 days. Each day, they received 10 food pellets, one of each of the 10 sizes, presented in a preassigned random order. Only one food pellet was placed in the food receptacle at a time. Once a rat retrieved a food pellet, another food pellet was placed in the receptacle. The behavior of a rat on retrieving a food pellet was entered into the computer by the experimenter, using the microswitches, as described above.

\section{Results}

Food-handling behavior. Food handling varied as a function of both distance from the refuge and size of the food pellet; the main effects of behavior, food-pellet size, and distance were all significant $(p s<.0001)$. At all distances, smaller pellets were usually eaten directly (eat), medium-sized pellets were eaten using a sitting posture near the food source (sit), and larger food pellets were carried to the cage before being eaten (carry). As distance increased, however, the incidence of carries decreased $[F(4,20)=15, p<.0001]$ and the incidence of sits correspondingly increased $[F(4,20)=6.98, p=.0011]$, whereas the incidence of eats remained the same.

Since travel distance decreased food carrying, the results were further analyzed to examine the effects of distance on responses to food pellets of different sizes. 

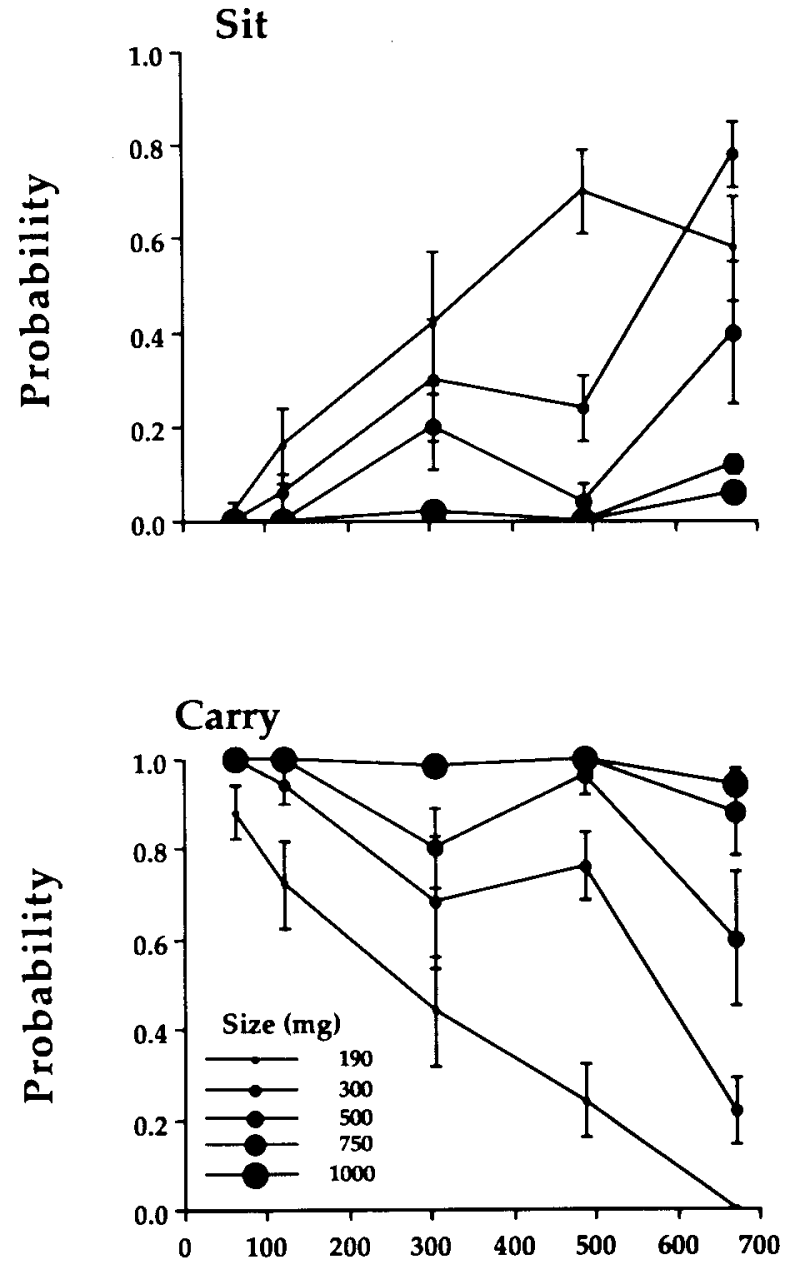

Distance (cm)

Figure 1. Effect of travel distance on the incidence of sits (top) and carries (bottom) with food-pellet sizes 6-10 (190-1,000 g). Note that the effect of distance is most pronounced on food-pellet sizes 6,7 , and 8 , for which the incidence of sits is directly proportional to distance and carries is inversely proportional to distance.

This analysis could only be made for food-pellet sizes 6-10, since smaller food pellets were seldom carried. The results shown in Figure 1 illustrate that there was a progressive decrease in the incidence of food carrying as a function of distance for food-pellet sizes 6,7 , and 8 . Sizes 9 and 10 were always carried. Thus, there were significant pellet $\times$ distance interactions for both sits $[F(36,180)=7.11, p<.0001]$ and carries $[F(36,180)=$ $9.41, p<.0001]$.

Food-handling time. A summary of the four foodhandling times is shown in Figure 2 for sizes 7-10, which were the only sizes that gave enough data with which comparisons could be made at all distances. All of the foodhandling times varied as a function of travel distance.

Carry time. The time taken to carry food pellets increased with increases in travel distance $[F(4,20)=43.9$;
Figure 2A]. In addition, carry times were shorter for larger food pellets than they were for smaller food pellets $[F(3,60)=2.61, p<.05]$.

Return time. Return times were slower for longer distances than for shorter distances $[F(4,20)=20.73$, $p<.0001$; Figure 2B] and were also slower following the consumption of large food pellets than following the consumption of smaller food pellets $[F(3,60)=0.61, p=$ .6]. Return times were also slower than carry times at all distances $[F(1,3)=18.4, p<.0001]$.

Eating time. As might be expected, the time required to eat larger food pellets was longer than the time required to eat smaller food pellets $[F(3,60)=1,228, p<.0001$; Figure $2 \mathrm{C}$ ]. Eating time, however, was also influenced by travel distance because the rats carrying food for longer distances consumed food more slowly than did the rats carrying food pellets for shorter distances [distance, $F(4,20)=2.98, p=.04$; pellet $\times$ distance interaction, $F(12,60)=2.37, p=.01]$.

Postprandial time. Overall, the rats were slower to leave the home cage after eating larger food pellets and were still slower to leave to travel longer distances [pellet size, $F(3,60)=5.2, p=.003$; distance, $F(4,20)=3.2, p=$ .03 ; see Figure 2D].

Relation between travel time and eating time. The relative influence of travel time and eating time on food carrying was estimated by comparing eating times of the smallest food pellet that was carried at least $50 \%$ of the time at each distance with the round-trip travel time for that food pellet at that distance. This comparison required normalizing the eating-time curve and then matching normalized points to the travel times at appropriate distances. These results are shown in Figure 3. The curve in Figure 3 suggests that if eating time exceeds return-trip travel time by about $20 \%$ to $40 \%$, a food pellet is likely to be carried.

There were some other features of motor behavior in the tests that require description. When returning to the home cage with food, the rats galloped at all distances. When advancing to the food, however, their motor behavior was related to distance. To traverse shorter distances, the rats usually left the cage cautiously and walked slowly to the food source. To travel longer distances, they left at a gallop but invariably stopped once or twice to look around before reaching the food.

\section{Discussion}

The results of Experiment 1 show that rat food-carrying behavior does vary as a function of travel distance. Overall, it appeared that when eating time exceeded returntrip travel time by about $20 \%$ to $40 \%$, the food was likely to be carried to the refuge for consumption. For example, size 6 food pellets took about $5 \mathrm{sec}$ to eat and were carried mainly at shorter distances where return-trip time was between about 3 and $5 \mathrm{sec}$; at longer distances, size 6 food pellets were hardly carried at all. Carrying of size 7 food pellets, which took between 10 and $15 \mathrm{sec}$ to eat, only decreased at the longest distances for which returntrip travel time exceeded $15 \mathrm{sec}$. The largest food pellets, 

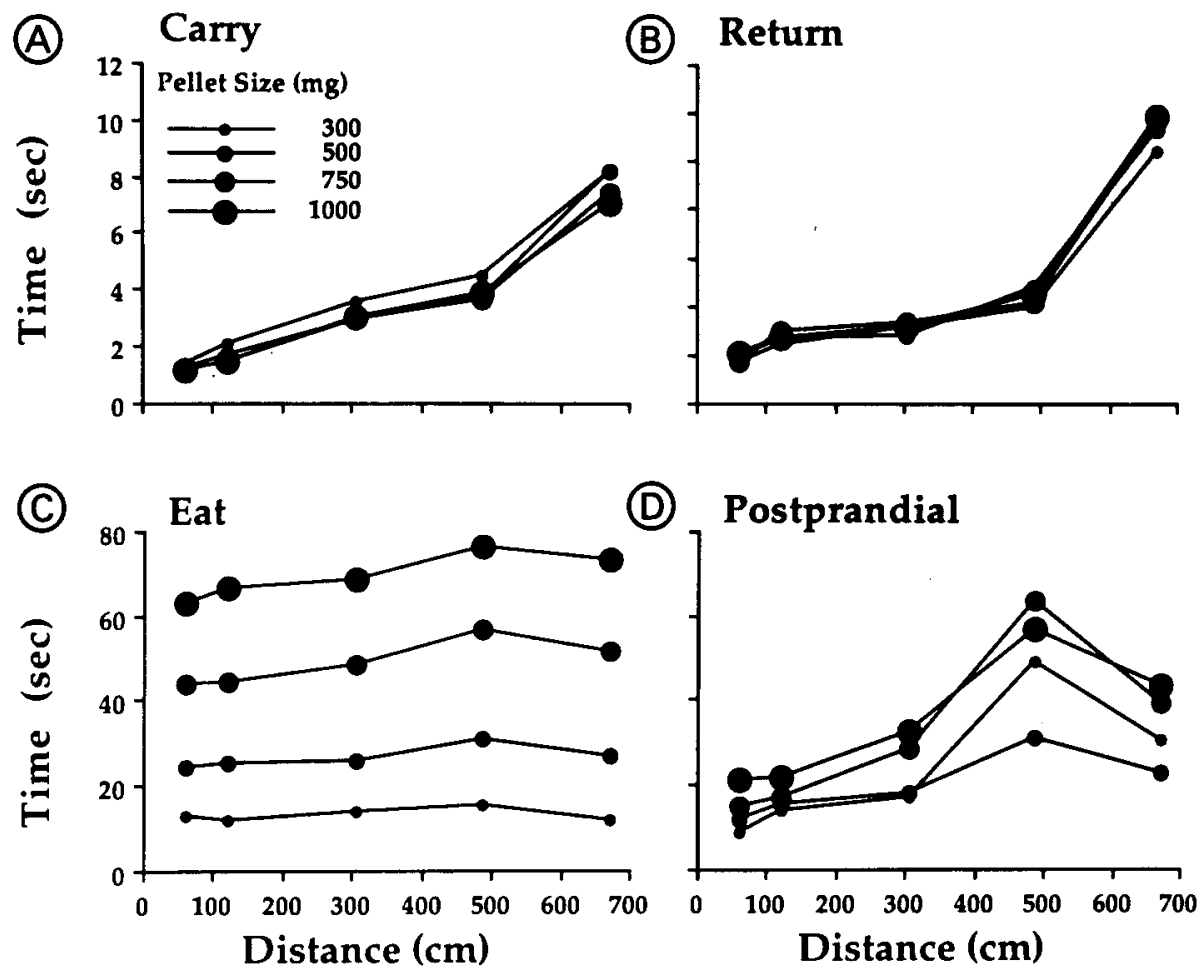

Figure 2. Effects of food-pellet sizes and travel distance on food-handling times. (A) Carry: time to return to cage with food. (B) Return: time between leaving home cage and reaching food pellet. (C) Eat: time to eat the food pellet. (D) Postprandial: time between finishing eating and leaving home cage. The results are arranged so that carry and return times can be compared; note that carry times are slower than return times.

sizes 9 and 10, were always carried, as might be expected since their eating time of over $40 \mathrm{sec}$ far exceeded roundtrip travel time of about $20 \mathrm{sec}$ at the longest distances. Thus, these results indicated that, for rats, as is the case for grey squirrels (Lima \& Valone, 1986; Lima, Valone, \& Caraco, 1985) and black-capped chickadees (Lima, 1985), travel distance does influence food carrying.

As has been reported previously for the rat, the approach time to the food was longer than the return time at all distances (Whishaw \& Tomie, 1989). Distance also had an effect on motor behavior. With the food located at the shorter distances, the rats approached the food cautiously with slow steps and low-slung posture; however, when it was located at longer distances, they galloped toward the food. What greatly reduced their approach time at the longer distances was not the speed with which they moved but the fact that they paused once or twice on each trip. These different motoric strategies are likely adaptive for detecting and evading predators. Caution in leaving the refuge and pausing on the approach to the food may enhance predator detection, whereas running without pause on the return trip reduces overall exposure time.

Two additional novel findings also emerged in Experiment 1 . Both eating time and postprandial time spent in the refuge were positively related to the distance that a rat had just traveled with a food pellet. We also confirmed that return-trip time is inversely related to the size of the food pellet that is being carried and time spent in the refuge after eating is directly related to the size of the food pellet that has just been consumed (Whishaw \& Gorny, 1991; Whishaw et al., 1990; Whishaw \& Tomie, 1989).

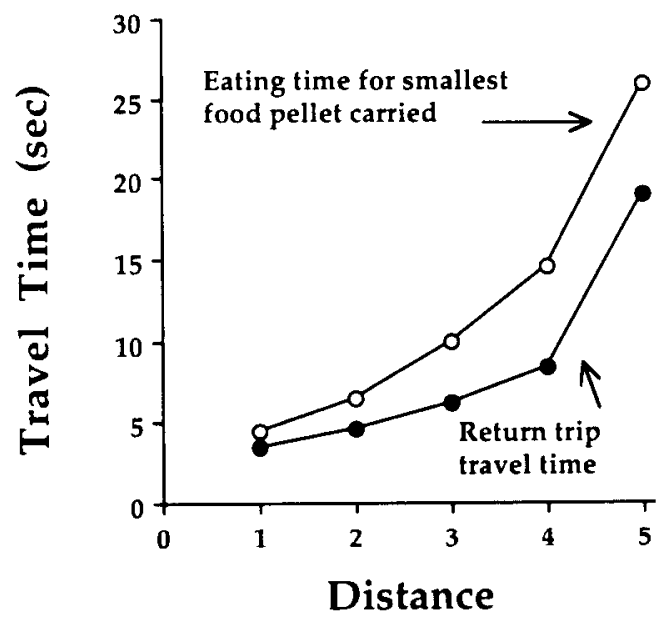

Figure 3. Relation between eating time and return-trip travel time for the smallest food-pellet size that was carried at least $50 \%$ of the time. Since the eating time and travel distances did not have the same scale, eating-time points were obtained from a regression curve of actual eating times. 
These food-pellet-size, travel-time, and distance effects on the various response times may indicate that the "value" accorded to food by the rat varies with the risk endured to obtain it (Whishaw et al., 1990; Whishaw \& Tomie, 1989). Thus, an animal that has just endured substantial risk or gained substantial reward would be less likely to hurry off on another foraging trip.

\section{EXPERIMENT 2 Effect of Travel Effort}

The results of Experiment 1 suggest that the relation between eating time and travel time is the underlying determining influence on decisions to carry food. If this is the case, then other variables that affect travel time should influence food carrying in the same way that changes in travel distance influence food carrying. The second experiment was designed to test this possibility. In Experiment 2, travel distance was held constant, but travel time was increased by successively reducing the width of the beam. This manipulation made the beam more difficult to cross and thus increased travel time.

\section{Method}

Animals. Twenty rats, selected from the groups used in Experiment 1, were randomly divided into four groups of 5 rats per group.

Apparatus. Four beams, $122 \mathrm{~cm}$ long, were used. They were $83,49,21$, and $9 \mathrm{~mm}$ wide. The animals traveled to the end of the beam in order to obtain a food pellet, which was placed on a small pedestal.

Pretraining. Each group of rats was assigned to one of the beam widths. The rats were then pretrained (as above) on the appropriate beam for 7 days.

Testing. The rats were tested for 6 days with 10 trials per day. Each day, a rat received each of the 10 differently sized food pellets, assigned in random order. When the rat had consumed one pellet, another pellet was placed at the food source. This continued until the rat had received all 10 sizes of pellets.

\section{Results}

Food-handling behavior. There was a significant effect of beam width on food-carrying behavior. As the beam was made narrower, the number of food pellets that were carried decreased and the number of times that the rats ate food at the food source correspondingly increased [carries, $F(3,16)=7.22, p=.003$; sits, $F(3,16)=13.8$, $p=.0001]$. There was no effect of beam width on eats $[F(3,16)<0.134, p>.05]$. The rats' behavior was also influenced by food-pellet size. At all distances, smaller pellets were usually eaten directly, medium-sized pellets were eaten using a sitting posture near the food source, and the largest food pellets were carried to the cage before being eaten. Thus, the main effects of behavior, food-pellet size, and beam width were all significant $(p s<.0001)$.

Since beam width influenced the incidence of food carrying, the results were further analyzed to examine the effects of width on food handling of individual food-pellet sizes. This analysis could only be made for food-pellet sizes 6-10, since an insufficient number of instances of these behaviors occurred with the smaller food pellets.
As is shown in Figure 4, as the width of the beam was decreased, there was a progressive increase in the size of food pellets that were carried. Size 10 pellets were mainly carried across all beam widths. The decrease in food carrying was paralleled by an increase in sits at the food source; thus, there were significant pellet $\times$ width interactions for both sits $[F(27,144)=4.60, p<.0001]$ and carries $[F(27,144)=5.6, p<.0001]$.

Food-handling time. A summary of approach and return times is shown in Figure 5 for sizes 7-10, which were the only sizes that gave enough data for which comparisons could be made at all beam widths. The rats did require longer times to traverse the narrowest beams $[F(3,20)=95.7, p<.001]$. They also took longer to approach the food than they took to return with a food pellet $[F(1,20)=9.95, p<.001]$. It is noteworthy that although carry and return times were different, the differences were very small. The mean overall carry time was $2.66 \mathrm{sec}$ and was only slightly faster than the return time of $2.97 \mathrm{sec}$. These travel times were not influenced by the size of the food pellets. The rats took longer to eat the larger food pellets $[F(3,12)=329.6, p<.0001]$, but eating time was not influenced by width of the beam that the animals traversed $[F(3,4)=1.75, p=.29]$. Fi-
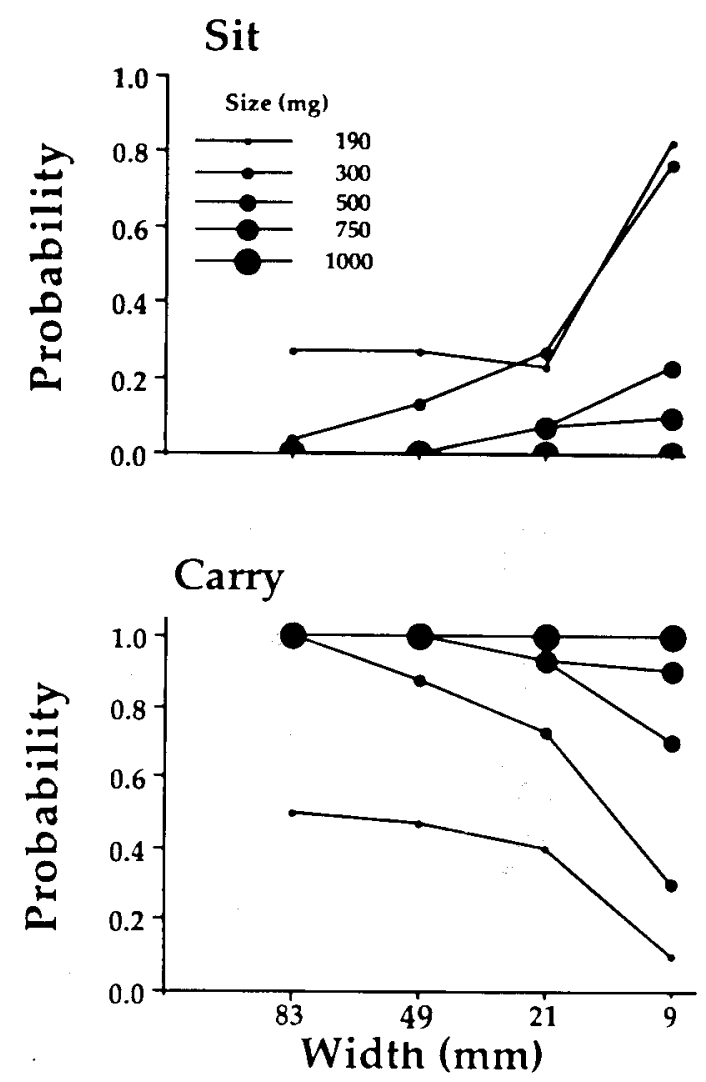

Figure 4. Effects of beam width and food-pellet size on incidences of sits and carries with food-pellet sizes 6-10 (190-1,000 g). Note that sits increased and carries decreased on the narrower beams and that this effect was most pronounced for pellet sizes 6-8. 


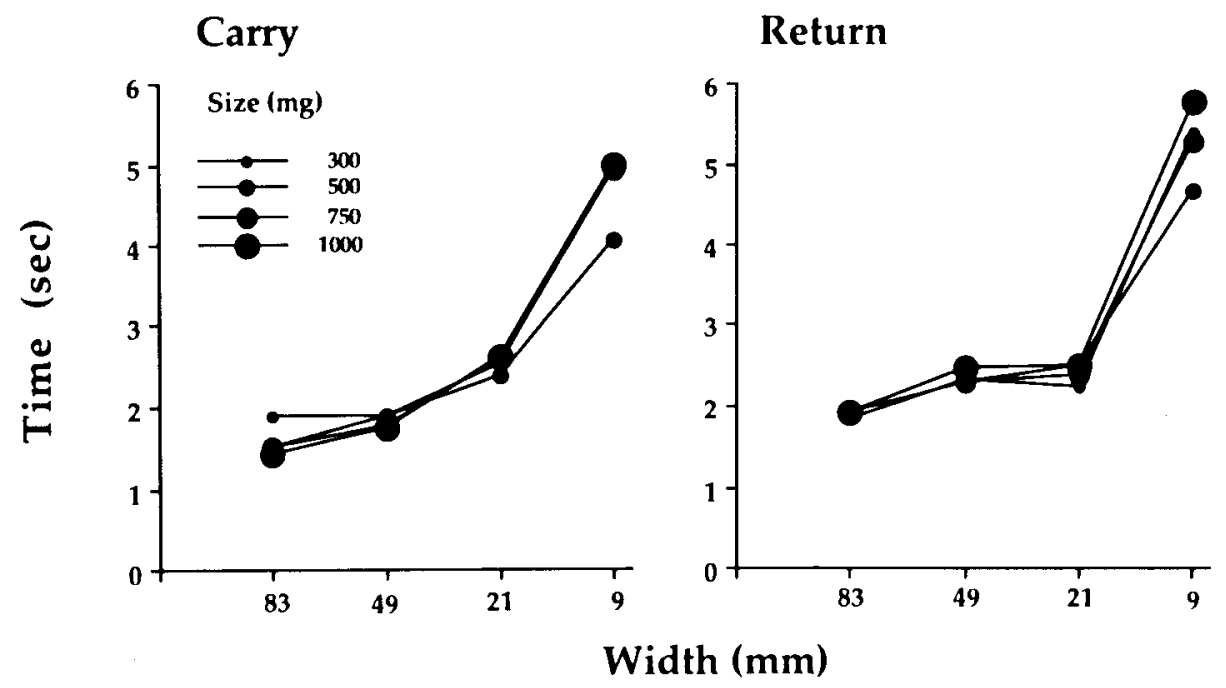

Figure 5. Effects of food-pellet size and beam width on carry time (time to return to the cage with food) and return time (time between leaving the home cage and reaching the food source).

nally, the rats spent longer periods of time in the home cage after eating the largest food pellets $[F(3,12)=16.2$, $p<.0002$ ], but beam width did not influence this postprandial cage time $[F(3,4)=1.3, p<.14]$.

Comparison of travel distance and travel time. To compare the effects of varying travel time by varying distance or by varying travel difficulty, the overall probability of carrying food was plotted against travel time for both conditions. The results are shown in Figure 6. The probability of food carrying across the first few comparisons was similar, but there was a wide discrepancy between the results obtained for the longest distance and for the narrowest beam. For a travel time of $10 \mathrm{sec}$ on the narrow beam, food carrying was attenuated to the same extent that it was for a travel time of $20 \mathrm{sec}$ on the longest beam.

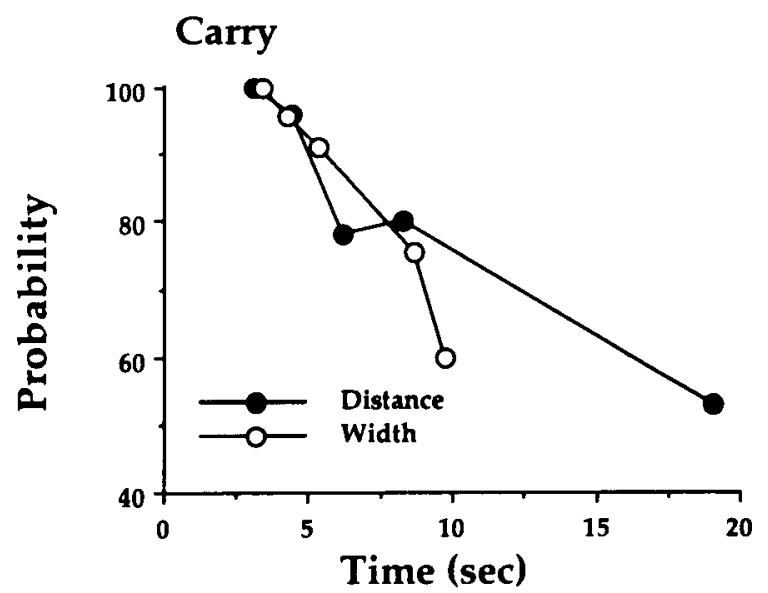

Figure 6. Probabllity of carrying food (mean of sizes 6-10 food pellets) as a function of travel time over different beam distances and beam widths. The results are normalized so that carry probability is expressed as a percent of carry probability in the first condition for both distance and width measures.

\section{Discussion}

Changing the difficulty of traversing the beam by making it narrow did increase travel time across the beam. There was also a corresponding reduction in food carrying as beam width was decreased. This result provided the opportunity to compare whether the relation between travel time and food carrying was the same for both distance and width manipulations. The result showed a very good relationship between the two conditions at some of the test points, but there was a striking divergence between the results obtained on the narrowest beam compared with those obtained on the longest beam. Hoarding probability decreased to about $60 \%$ at a round-trip time of $10 \mathrm{sec}$ on the narrowest beam but did not decrease to this level until a round-trip time of $20 \mathrm{sec}$ was reached on the longest beam.

The comparison between the beam distance and width experiments suggests that the influence that travel difficulty has on food carrying is at least partly independent from the effect that travel time has on food carrying. This independent influence could be risk of falling. The rats did have trouble balancing on the narrow beams, and they also had problems turning around on the narrow beams to initiate a return trip. Furthermore, on many training trials and on some test trials, they slipped on the beams and had to be rescued. Thus, it is possible that risk of falling may oppose the influence of risk of predation in influencing food-carrying behavior.

\section{EXPERIMENT 3 Influence of Light, Dark, or Cat Odor}

The results of Experiment 2 suggested that factors other than just travel time and food-consumption time influence food carrying. The purpose of Experiment 3 was to examine the effect of other forms of stress or threat on food 


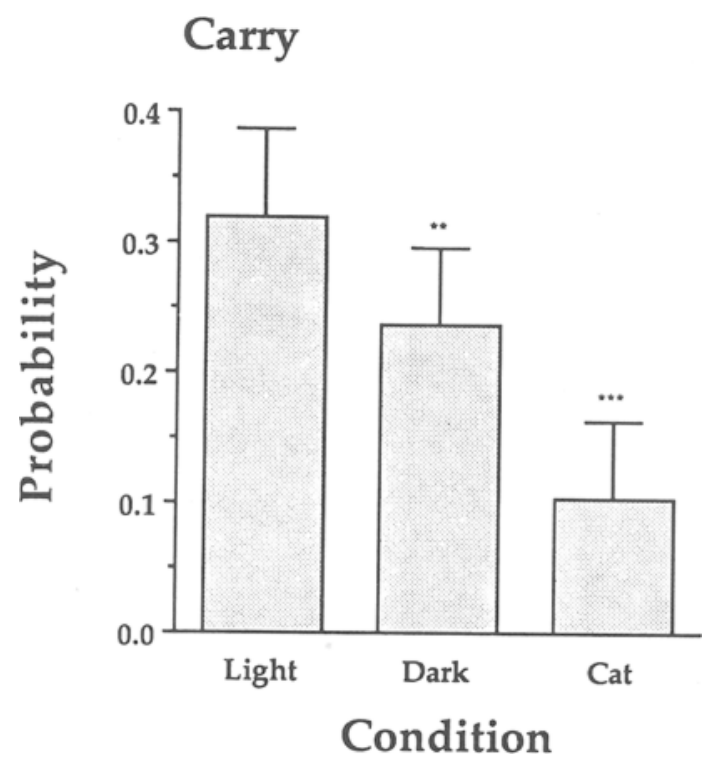

Figure 7. Carry probability (of all 10 pellets) in three test conditions. Note that the incidence for carries decreased during the dark condition and that most rats failed to leave the refuge in the cat condition, producing a further decrease of carries. (Newman-Keuls tests: $* *=p<.01$, difference between light and dark conditions; $* * *=$ $p<.01$, difference between cat and both light and dark conditions.)

carrying. Food carrying was examined in three different situations: in the light, in the dark, and when a cat was present in the room. It was expected that food carrying would decrease in the dark since the animals are less obviously exposed in the dark (Whishaw et al., 1990). It was also expected that food carrying might increase in the presence of the odor of a cat since this odor is perceived as threatening by rats (Blanchard \& Blanchard, 1989).

\section{Method}

Animals and Pretraining. The five groups of rats used in Experiment 1 were used in the tests of Experiment 3. Each group was tested in the distance condition on which it had been pretrained in Experiment 1.

Tests. The rats received three tests. (1) Light: in the light test, the rats received the same test described in Experiment 1 . Each group was given one of each of the 10 food pellets, at the distance appropriate for that group. (2) Dark: the same procedure was used for the dark test except that the room lights were turned off so that the room was completely dark. The computer screen was faintly illuminated so that data could be collected, but the screen faced away from the test apparatus. The dark test was given 1 day following the light test. (3) Cat: the cat test was given in the light as described above, with the exception that a cat, housed in a wire cage, was placed on the floor at the end of the beam beside the tester where it could not be seen from the refuge.

\section{Results}

A summary of overall hoarding scores obtained in the light, dark, and cat tests is shown in Figure 7 . The rats carried fewer food pellets in the dark condition than they did in the light condition. When the cat was present, food carrying was reduced even further, with 18 of 25 rats fail- ing to leave the refuge at all; these animals received scores of zero for food carrying. For the 7 rats that did leave the home cage, food carrying was similar to that observed in the light condition. These main points were confirmed by statistical analyses. There were significant main effects of test condition $[F(2,40)=26.6, p<.0001]$, travel distance $[F(4,20)=3.8, p<.019]$, and pellet size $[F(9,180)=162.3, p<.0001]$. Follow-up NewmanKeuls tests indicated that the light, dark, and cat conditions were also different from each other $(p<.01)$. As might be expected, all of the interactions were also significant $(F \mathrm{~s}>2.2$, ps $<.05)$.

To evaluate the relation between eating time and food carrying in light and dark conditions, carrying probability scores were summed across distances and plotted against food-pellet eating times. These results are shown in Figure 8. In the light, the probability of carrying food increases with eating time. A similar effect was obtained in the dark; however, it is apparent that the main effect of reducing illumination was to shift the food-carrying probability curve to the right such that the animals only carried the largest food pellets.

\section{Discussion}

When the rats were tested in the dark, they continued to carry food to the refuge, but they carried more food at shorter distances than at longer distances, and thus the overall incidence of food carrying was greatly reduced. Thus, when food-carrying probability was plotted against eating time, it was clear that the curve generated in the light condition was shifted to the right in the dark condition. Therefore, the results suggest that illumination has an influence on food carrying that is independent of the effects of food size and travel distance.

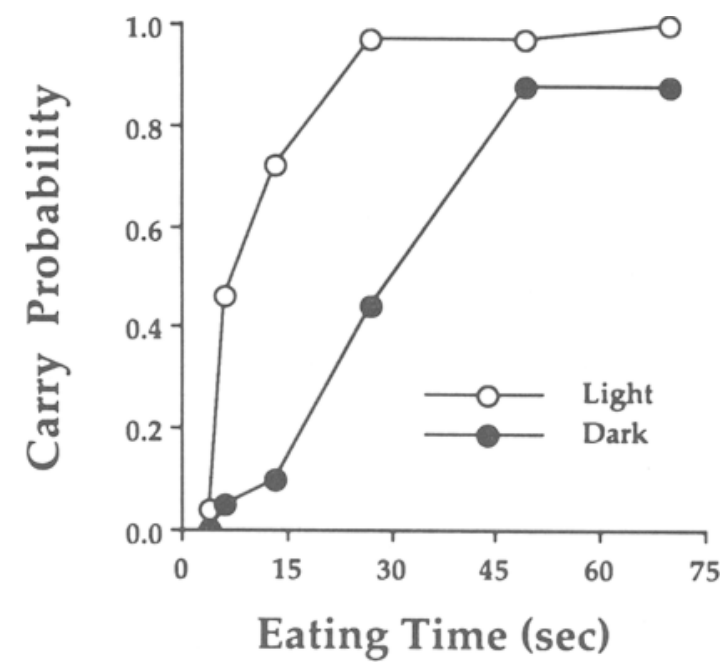

Figure 8. Relation between eating time and the probabillty of carrying food in light and dark tests. Note that, in both conditions, there is a close relation between eating time and probability of carrying food but, for the dark condition, the curve is shined to the right, relative to the curve for the light condition. 
We were surprised initially by the results of the catodor study. We had expected that in the presence of potential predatory threat, the rats would have been more likely to carry food. Most of the rats refused to leave the refuge cage and approach the food, however. Thus, the cat odor, rather than influencing food carrying, blocked foraging. Additionally, this effect was quite long-lasting. We attempted to test the rats on the following day (after the cat was absent from the room for $24 \mathrm{~h}$ ), and most of the animals would still not leave the cage.

The finding that cat odor inhibits exits from a home base is consistent with the report of Blanchard and Blanchard (1989). With respect to foraging, the results provide an additional testament to the flexibility of the behavioral responses that rats display while foraging. With results in hand, we realized that if the rats had simply responded by carrying more food, they would have had to carry smaller food pellets and thus would not only have increased the time they were exposed but would have risked themselves for relatively small food pellets. Thus, in the presence of threat of the kind that a cat odor signals, it would seem to be most adaptive to delay foraging until the threat had passed.

\section{EXPERIMENT 4 Effect of Food Quantity and Size}

In previous studies of rat food carrying, we have found that when a number of food pellets are provided at the food source, rats will carry large- and intermediate-sized food pellets, often attempting to take more than one food pellet on a trip. They did not attempt to carry the smallest food pellets (Whishaw et al., 1990; Whishaw \& Tomie, 1989). In these studies, only a few food pellets were available, however. The final experiment of this series had two objectives. The first objective was to determine how rats would respond to a very large number of small food pellets at the food source. The second part of the experiment was designed to determine what the rats would do when presented simultaneously with a large number of small and large food pellets.

\section{Method}

Animals. The five groups of rats used in Experiment 1 were used. Pretraining. The rats were tested on the beam used in Experiment 1 , with each group given food pellets at the distance appropriate for that group. One day of pretraining was given. On that day, a dish containing 1,000 20-mg food pellets was located at the end of the beam, and the rats received one trial, which ended when they returned to the refuge.

Test. The rats were tested on each of 20 successive days. On Days 1-10, a dish containing one of the 10 sizes of food pellets was located at the end of the beam. The order of presentation of sizes was assigned according to a predetermined randomization procedure. Each rat was allowed one trial, which consisted of a trip to the end of the beam and which ended when the rat returned to the refuge cage. The time spent eating was recorded, and the number of food pellets eaten was counted. On Days 11-20, there were two dishes located at the end of the beam. One dish was filled with one of the 10 sizes of food pellets; the second dish always contained size $10(1,000 \mathrm{mg})$ food pellets. The choice of food pellets, time spent eating at the food source, and the number of food pellets eaten were recorded. A trial ended when a rat returned to refuge, whether or not it carried a food pellet.

\section{Results}

A summary of the results is shown in Figure 9. Overall, there were large differences that depended upon the test, food-pellet size, and distance. The analysis of variance also indicated that there were significant differences in eating time and number of food pellets eaten between the choice and no-choice conditions $\left[F_{\mathrm{s}}(1,4)>100\right.$, $p s<.0001]$. In view of this difference, separate analyses were done for each condition.

No-choice test. When smaller food pellets were present, large numbers were eaten at the food source, and the number eaten was directly related to travel distance. Consequently, the time spent at the food source was also directly related to travel distance. If intermediate-sized food pellets were present, some were eaten at the food source, especially at the longer distances, but, generally, the rats would pick up a number of food pellets at once and carry them to the refuge. When larger food pellets were present, they were nearly always carried to the refuge from all distances.

These results were confirmed by analyses that showed that there was a significant effect of food-pellet size for eating time $[F(9,180)=11.0, p<.0001]$ and number of pellets consumed $[F(9,180)=23.5, p<.0001]$; there was also a significant effect of travel distance on both eating time $[F(4,20)=6.7, p=.0014]$ and number of pellets eaten $[F(4,20)=5.8, p=.0029]$. Finally, there was a significant interaction between food-pellet size and distance for number of pellets eaten $[F(36,180)=1.4$, $p<.0001]$, but not for the time spent eating.

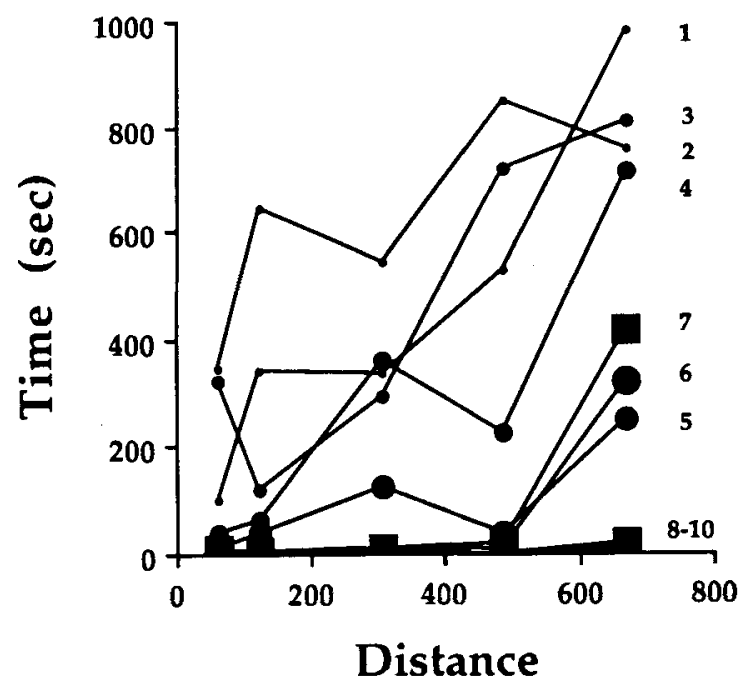

Figure 9. Time spent eating at food sources located at different distances as a function of food-pellet size. Numbers to the right identify the curves for food pellets of each of the different sizes. Note that eating time is longer for smaller food pellets than for larger food pellets, and eating time increases with travel distance. 
Choice test. In the choice test, the rats always selected a large food pellet and carried it to the refuge. Thus, there was a large significant choice effect $[F(1,2)=45.3$, $p<.0001]$. However, there were no significant effects for time, number of pellets eaten, or distance, and there were no significant interactions.

\section{Discussion}

Experiment 4 demonstrated that if large food pellets were present at the food source, they would be preferentially selected and carried to the refuge. If only small food pellets were present, however, the rats would stay and eat large numbers of them. Furthermore, the farther the food was from the refuge, the longer the rats would stay and eat. Thus, the rats might stay at the most distant food source almost five times longer than at the closest food source.

The results show that there is a lower limit on the size of food pellets that rats will carry. The rule appears to be: food sizes that are not carried when they are presented individually will not be carried even if large quantities are available. Rather, the rats will spend a very long time at the food source eating them.

Given our predisposition to believe that rats find remaining in the open both risky and aversive, we were initially surprised by the length of time that they would stay eating at the food source. The results also suggest that the upper limit that rats will remain eating in the open is quite high, between 5 and $10 \mathrm{~min}$. This upper limit may be the time required to obtain a complete "meal," but we did not investigate this. With results in hand, this behavior is sensible. If the rats periodically interspersed returns to the refuge with bouts of eating, the travel time would increase overall exposure time but would constitute time that they were not consuming food. Thus, although staying at the food source for a long period of time might pose risks, exposure time is still less than would occur with interspersed eating and periodic returns to the refuge. Arguably, the rats also may be less noticeable to predators when they are eating at the food source than when they are running back and forth.

\section{GENERAL DISCUSSION}

The results of this study suggest that a variety of independent factors influence rat food-carrying behavior, including food size, eating time, travel distance, travel risk, predation risk, and food availability. Furthermore, rats appear to have a variety of behavioral responses that they can use flexibly in various foraging conditions, including a number of eating, approach, and carrying responses, the speed of which can be varied as situations change. In general, these results are consistent with optimal foraging theory, which suggests that individual foraging acts will reflect a tradeoff between food-gathering behavior and predator avoidance behavior. In addition, however, the results show that rats respond adaptively to a surprising array of environmental events, some of which have additive or competing influences. Although very little is known about the neural mechanisms that mediate this rich foraging behavior, the present results suggest that the rat provides an excellent laboratory subject for experimental investigations of the neural control of foraging.

The results confirm previous work in showing that food size and anticipated eating time influence food carrying in the rat. In addition, the results show that travel distance influences food carrying, thus confirming results obtained with grey squirrels (Lima \& Valone, 1986) and black-capped chickadees (Lima, 1985). The results also show that other factors influence food carrying including travel difficulty, lighting conditions, predator odor, and availability of food. Since many of the same conditions also influence an animal's susceptibility to predation, the main findings can be considered within Lima and Valone's (1985) multifactor optimal foraging theory.

In a previous study using rats, we had found that food carrying was not influenced by travel distance (Whishaw \& Tomie, 1989). The present results show that the distances used in that study were probably too short to produce obvious effects. Here, we found that there was a systematic reduction in the size of carried food pellets as distance was increased. The critical factor in producing the result appeared to be that round-trip travel time had to exceed eating time by about $40 \%$. This result is consistent with the notion that there is a tradeoff between the amount of time exposed to potential predation and the amount of food that can be consumed, such that if overall exposure time is reduced by food carrying, animals will carry. We were unable to evaluate whether there is some upper limit on the size of a food pellet that will be eaten in the open or an upper limit to the distance that a rat will carry food. The longest distance used in the present study was the length of the test room. Nevertheless, it is likely that once a piece of food reaches the size that it comprises a meal in itself, it will be carried irrespective of the distance to a refuge. This question could be examined in future studies.

We were interested in determining whether or not time estimates can simply account for most decisions to carry food. Therefore, we manipulated travel time by increasing travel difficulty and by increasing travel distance. It was our expectation that if time was the critical variable underlying food-carrying decisions, we would obtain similar time/carry functions for the distance and difficulty manipulations. The functions were not the same, however. For the condition in which a rat had to walk on the narrowest beam, we found that the rats were much less likely to carry food than was predicted by the time values obtained on the distance experiments. Thus, travel time does not appear to account for the food-carrying results in any simple way. This suggests to us that the beamwalking task introduced an additional influencing factor. The rats did have difficulty walking on the beam, especially during pretraining: they often slipped from it as they walked or as they attempted to turn around with the food. We think that it is possible that "travel risk" represented by risk of falling becomes a factor in influencing foodcarrying decisions. Thus, travel time and travel risk may 
be additive. Whatever the correct interpretation, the results are clear in indicating that food carrying cannot be simply reduced to a function of travel time.

Further evidence to suggest that carrying decisions cannot be simply accounted for by the relation between eating time and travel time came from an experiment in which lighting was varied. We found that when comparisons of food carrying were made in lighted versus dark conditions, the rats continued to respond to food size and travel distance, but they showed much less food carrying in the dark. As a result, when food carrying was plotted against eating time for food pellets of different sizes, the curve for the dark was shifted to the right, relative to the curve for the light. That is, food pellets had to be much larger and located much closer to the refuge if they were to be carried in the dark condition. We attempted to see if we could find a simple rule that would predict food carrying in both light and dark conditions. There was no obvious relation, because, in the light, the rats carried food if eating time exceeded round-trip travel time by about $40 \%$ at all distances, whereas in the dark, eating time had to exceed round-trip travel time by a much greater amount for carrying to occur. Despite the difficulties in predicting food carrying in both light and dark conditions, the results are generally consistent with the tradeoff hypothesis of food carrying. If it is assumed that rats are at less risk to predation in the dark than in the light, they should be more likely to eat larger items at the food source in the dark than in the light, as was found.

In designing the experiment in which a cat was introduced into the test room, we had assumed that rats would respond as if they were subject to greater risk and might therefore carry smaller food pellets at all travel distances. We were surprised that most rats refused to leave the refuge to approach the food. Although this result initially appeared counterintuitive, upon reconsideration it does make sense. First, cat odor seems to have a rather general effect in blocking rat excursions from a refuge (Blanchard \& Blanchard, 1989). Second, if cat odor does signal the potential presence of a predator to a rat, then remaining in the refuge would be a more adaptive response than attempting to forage. In light of this argument, our expectation that the rats might carry smaller food pellets is particularly misplaced since they would be increasing their risk to predation for rather minimal benefit. Finally, it is possible to speculate that rats might perceive the predator as a temporary risk and one which could be adequately avoided by delaying foraging excursions.

A persuasive demonstration in favor of optimal foraging theory was obtained when a large supply of food was made available at the food source. If the food consisted of small food pellets, the rats remained eating for very long periods of time, particularly at the farthest distances. This behavior did not simply reflect the availability of food, since if the food pellets were large, or if the rats had an opportunity to choose a large food pellet, the food was immediately carried. We had initially thought that when faced with a supply of small food pellets, the rats might either begin to carry a mouthful of food or intersperse bouts of eating with returns to the refuge. When we calculated the net benefits of traveling versus eating at the food source, however, we found that it is unlikely that the rats could carry enough food to make a trip worthwhile. Thus, traveling would simply add exposure time and energy expenditure without providing sufficient benefits to override remaining to eat at the food source. Thus, our calculations supported the rats' decision to eat at the food source.

Overall, the results of these experiments provide strong support for optimal foraging theory. Even though rats display a surprising variety of behaviors in different foraging conditions, in every case, their selection of a behavior appears to maximize food intake while minimizing the amount of time they are exposed to potential risk. In addition to highlighting the richness of the rats' adaptive behavior, the results show that there is no simple underlying rule, such as the relation between eating time and travel time, that determines whether food is carried. Rather, behavior appears to be orchestrated by a number of hierarchically arranged priorities. For example, although a relation between eating time and travel time appears predictive in daylight conditions, the relationship can be dramatically shifted in favor of less food carrying either by reducing ambient lighting or by increasing travel difficulty. It is probable that other influences (e.g., the kind of potential predator, the animals' familiarity with the foraging environment, level of food deprivation, etc.) can also modulate food-carrying behavior. Future work may usefully consider how the various influences on food carrying are prioritized.

\section{REFERENCES}

BLANCHARD, R. J., \& BlanChaRd, C. D. (1989). Attack and defense in rodents as ethoexperimental models for the study of emotion. Progress in Neuro-Psychopharmacology \& Biological Psychiatry, 13, S3-S14.

EWER, R. F. (1971). The biology and behavior of a free-living population of black rats (Rattus rattus). Animal Behavior Monographs, 4, 1226-1274.

KREBS, J. R., MCCleery, R. H. (1984). Optimization in behavioral ecology. In J. R. Krebs \& N. B. Davies (Eds.), Behavioral ecology (2nd ed., pp. 91-121). Sunderland, MA: Sinauer.

Lma, S. L. (1985). Maximizing feeding efficiency and minimizing time exposed to predators: $A$ trade-off in the black-capped chickadee. Oecologia (Berlin), 66, 60-67.

LIMA, S. L. (1987). Distance to cover, visual obstructions, and vigilance in house sparrows. Behavior, 102, 231-238.

Lima, S. L., VALONE, T. J. (1986). Influence of predation risk on diet selection: A simple example in the grey squirrel. Animal Behaviour, 34, 536-544.

Loma, S. L., Valone, T. J., \& Caraco, T. (1985). Foraging-efficiencyrisk trade-off in the grey squirrel. Animal Behaviour, 33, 155-165.

MCNamara, R. K., Whishaw, I. Q. (1990). Blockade of hoarding in rats by diazepam: An analysis of the anxiety and object value hypotheses of hoarding. Psychopharmacology, 101, 214-221.

Phel.Ps, M. T., \& Roberts, W. A. (1989). Central-place foraging by Rattus norvegicus on a radial maze. Joumal of Comparative Psychology, 103, 326-338. 
Valone, T. J., LimA, S. L. (1987). Carrying food items to cover for consumption: The behavior of ten bird species feeding under the risk of predation. Oecologia (Berlin), 71, 286-294.

WhISHAW, I. Q. (1990). Time estimates contribute to food handling decisions by rats: Implications for neural control of hoarding. Psychobiology, 18, 460-466.

Whishaw, I. Q., \& GoRnY, P. B. (1991). Postprandial scanning by the rat (Rattus norvegicus): The importance of eating time and an application of "warm-up" movements. Joumal of Comparative Psychology, $105,39-44$

Whishaw, I. Q., Gorny, B. P., Dringenberg, H. C. (1991). The defensive strategies of foraging rats: A review and synthesis. Psychological Reports, 41, 185-205
Whishaw, I. Q., OdDIE, S. D. (1989). Qualitative and quantitative analyses of hoarding in medial frontal cortex rats using a new behavioral paradigm. Behavioural Brain Research, 33, 255-266.

Whishaw, I. Q., Oddie, S. D., McNamara, R. K., Harres, T. L., \& Perry, B. S. (1990). Psychophysical methods for study of sensorymotor behavior using a food-carrying (hoarding) task in rodents. Journal of Neuroscience Methods, 32, 123-133.

Whishaw, I. Q., Tomie, J.-A. (1989). Food-pellet size modifies the hoarding behavior of foraging rats. Psychobiology, 17, 93-101.

(Manuscript received January 16, 1991; revision accepted for publication May 10, 1991.)

\title{
Notices and Announcements
}

\author{
21st Annual Meeting of the Society for Computers in Psychology \\ San Francisco, California \\ November 21, 1991
}

The 21st Annual Meeting of the Society for Computers in Psychology will be held at the Hyatt Regency Hotel at Embarcadero Center in San Francisco on November 21, 1991, the day before the annual meeting of the Psychonomic Society. The meeting will include presentations, discussions, tutorials, and times for software and hardware demonstrations. All areas of psychology are featured, including research, education, clinical practice, and industrial applications.

For further information regarding the conference, contact William L. Palya, Department of Psychology, Jacksonville State University, Jacksonville, AL 36265 (BITNET address FWLP@JSUMUS, phone (205) 7825641, FAX (205) 782-5680).

\section{2nd Annual Meeting of the Psychonomic Society \\ San Francisco, California \\ November 22-24, 1991}

The 32nd Annual Meeting of the Psychonomic Society will be held in San Francisco, November 22-24, 1991. The meetings will begin Friday morning and continue until Sunday at noon. The headquarters hotel will be the Hyatt Regency San Francisco at Embarcadero Center.

The program and hotel reservation cards have been mailed to members and associates. A copy of the program will be published in the November issue of the Bulletin of the Psychonomic Society.

For further information, please contact the secretary-treasurer of the Society: Cynthia H. Null, P.O. Box 7104, San Jose, California 95150-7104 (telephone: 415-604-1260). 